\title{
Persistent physical exercise raises the plasma concentration of vasohibin-1 in patients with peripheral vascular disease
}

\author{
Yasuhiro Suzuki ${ }^{1}$, Osamu Ito ${ }^{2}$, Masahiro Kohzuki ${ }^{2}$, Masataka Ichiki ${ }^{3}$ and Yasufumi Sato ${ }^{*}$ \\ ${ }^{1}$ Department of Vascular Biology, Institute of Development, Aging and Cancer, Tohoku University, Japan \\ ${ }^{2}$ Department of Internal Medicine and Rehabilitation Science, Tohoku University Graduate School of Medicine, Japan \\ ${ }^{3}$ Department of Surgery, Japan Railway Sendai Hospital, Japan
}

\begin{abstract}
Vasohibin-1 (VASH1), an endothelium-derived angiogenesis inhibitor, has unique functions of promoting stress tolerance and survival of endothelial cells. The expression of VASH1 decreases with aging, and this reduction may worsen vascular diseases such as diabetic vascular complications and atherosclerosis. Here we determined the plasma level of VASH1 in normal volunteers and patients with peripheral vascular disease. The plasma VASH1 concentration tended to be higher in males than in females in the normal volunteer group, and acute exercise did not alter this level, indicating that this concentration remained steady. Patients with peripheral vascular disease were older, and their plasma VASH1 concentrations tended to be lower. When those patients received physical exercise therapy over a 6-month period, their plasma VASH1 level increased significantly. This is the first demonstration that physical exercise can modulate the plasma VASH1 level in elderly patients with peripheral vascular disease.
\end{abstract}

\section{Introduction}

The vascular endothelium covers the entire luminal surface of blood vessels, organizes the interface between the blood and underlying tissues, and functions to control vascular tone, blood clotting, transport of various substances across the vascular wall, adhesion and transmigration of leukocytes, and so forth. Thus, the structural and functional integrity of the vascular endothelium is essential for the maintenance of vascular health [1]. Cardiovascular diseases (CVDs) are the major cause of death in industrialized countries. It is well accepted that aging is the critical risk factor of CVDs, as the prevalence of CVDs is significantly increased in the older population [2]. Aging is associated with the progressive deterioration of the structure and function of all types of cells, including vascular endothelial cells (ECs). Indeed, ECs receive cumulative cellular damage during aging, and this age-dependent impairment of the vascular endothelium is a key factor contributing to age-related vascular diseases [3].

Physical exercise is reported to play a significant role in the prevention of CVD and to reduce the mortality risk [4]. It is believed that the beneficial effects of physical exercise result from a reduction in traditional risks such as high blood pressure, elevated serum lipids, and high blood glucose and/or excessive body weight. However, an epidemiological study claimed that modification of those risks accounted for less than $50 \%$ of the improvement due to physical exercise, and thus the entire mechanisms underlying the beneficial effect of physical exercise on the vasculature are not yet known [5].

In order to maintain vascular integrity, the vascular endothelium should have self-defense systems. We previously reported that vasohibin-1 (VASH1) is one such systems [6]. VASH1 is originally isolated as an endothelium-derived angiogenesis inhibitor acting as a negative-feedback regulator [7]. However, our subsequent analysis revealed that VASH1 has an additional function of elevating the stress resistance of ECs by increasing the expression of superoxide dismutase
2 (SOD2) and SIRT1 in ECs [8]. Thus, VASH1 provides a novel link between inhibition of angiogenesis and tolerance to vascular stress [6].

Although VASH1 synthesis can be augmented in ECs transcriptionally by angiogenic stimuli such as vascular endothelial growth factor (VEGF), or post-transcriptionally by HUR, an RNAbinding protein, the basal synthesis of VASH1 protein in ECs is down-regulated during the replicative senescence of ECs due to an increase in the level of a certain microRNA, namely, miR-22 [8-10]. In addition, our studies with vash1-targeted mice revealed that the decreased expression of VASH1 promotes the progression of vascular diseases such as diabetic nephropathy and atherosclerosis [10,11]. Thus, maintenance of the VASH1 level is desirable for vascular health, especially in the elderly. The aim of the present study was to determine the level of VASH1 in both male and female subjects and to test whether or not physical exercise could modulate it.

\section{Experimental procedures}

This study was approved by the ethics committees of both Tohoku University School of Medicine and Japan Railway Sendai Hospital. Informed consent was obtained from all the participants. Participants were 75 normal volunteers, 22 males and 53 females; and 29 patients with peripheral artery disease (PAD), 25 males and 4 females. Plasma samples were collected, and their VASH1 levels were determined by

Correspondence to: Yasufumi Sato, Department of Vascular Biology, Institute of Development, Aging, and Cancer, Tohoku University, 4-1, Seiryo-machi, Aoba-ku, Sendai 980-8575, Japan. Tel: +81-22-717-8528, Fax: +81-22-717-8533; E-mail: yasufumi.sato.b3@tohoku.ac.jp

Key words: vasohibin-1, exercise, peripheral vascular disease

Received: November 24, 2016; Accepted: December 19, 2016; Published: December 22, 2016 
ELISA. The ELISA for VASH1 was described previously [12].

\section{Acute exercise}

Eight normal volunteers (8 males) were subjected to acute exercise, and the difference in their plasma VASH1 levels before and after the acute exercise was examined. Loaded acute exercise was done by symptom-limited cardiopulmonary exercise testing (CPET) on a bicycle ergometer (Q STRESS; Nihonkoden, Tokyo, Japan) according to a ramp protocol with a workload increment of $15 \mathrm{~W} / \mathrm{min}$.

\section{Physical exercise therapy}

A physical exercise therapy was applied to 18 patients with PAD (15 males and 3 females) for 6 months. The daily loaded physical exercise was a 30 -min walk twice a day according to the guidelines in a supplement to the Journal of Vascular Surgery [13].

\section{Statistical analysis}

The difference in the plasma VASH1 level between male and female normal volunteers was tested for statistical significance by performing Student's $t$-test; and the change in plasma VASH1 after acute exercise or 6-month physical exercise, by using the paired $t$-test. Results were considered to be statistically significant if p-values were less than 0.05 .

\section{Results}

The VASH1 protein is composed of 365 amino acids, and the calculated molecular weight is $44 \mathrm{kDa}$. This protein is proteolytically processed into 2 truncated forms after its secretion. VASH1 protein of $36 \mathrm{kDa}$ is an $\mathrm{N}$-terminally truncated form retaining the anti-angiogenic activity; whereas the $29-\mathrm{kDa}$ VASH1 protein is truncated at both $\mathrm{N}$-terminal and C-terminal regions and has no anti-angiogenic activity $[14,15]$. The ELISA that we used could detect bioactive $44-\mathrm{kDa}$ and $36-$ $\mathrm{kDa}$ forms but not the inactive $29-\mathrm{kDa}$ form (Figure 1).

By using this ELISA, we assayed the plasma VASH1 concentration of normal volunteers and patients with PAD. As shown in figure 2, patients with PAD were older, and their plasma VASH1 tended to be lower than that in the normal-volunteer group. The plasma VASH1 level of male volunteers tended to be higher than that of the female ones, although the difference was not statistically significant (Figure 3). When we subjected normal volunteers to acute exercise and compared their plasma VASH1 concentrations before and after the exercise, we could not observe any changes (Figure 4).

Finally, we had PAD patients perform daily physical exercise. As we could not obtain all samples taken before the exercise, we compared levels in samples taken after 1 month and 6 months of exercise. The results showed that daily physical exercise significantly increased the plasma VASH1 concentration (Figure 5).

\section{Discussion}

This is the first demonstration that physical exercise could increase the plasma VASH1 concentration in patients with peripheral vascular disease. Importantly, this increase could be achieved in the elderly, although their plasma VASH1 levels were lower. The plasma VASH1 concentration tended to be higher in males than in females in the group of normal volunteers. We speculate that this tendency might have been due to the difference in physical activities between male and female. As it has been demonstrated that the expression of VASH1 decreases with age and that a decreased expression of VASH1 can be a risk factor for the progression of vascular diseases, we believe this increase in VASH1 to be beneficial for cardiovascular health $[10,11]$.

As mentioned earlier, the synthesis of VASH1 can be induced transcriptionally by angiogenic stimuli or post-transcriptionally by the RNA-binding protein HUR. In addition, VASH1 is proteolytically degraded and inactivated after its secretion [14,15]. The ELISA that we used in the present study detected the biologically active forms of VASH1. Consequently, it is not clear at this time whether aerobic exercise increased the synthesis or decreased the degradation of VASH1, and this point should be clarified in a future study.

An important issue of physical exercise therapy is how to determine its optimal amount, intensity, and duration. Several biomarkers have been examined to analyze the effect of such therapy and all the parameters related to inflammation and metabolism are known to be decreased after physical exercise therapy [5,16-18]. In contrast, the VASH1 level was increased after the physical exercise therapy. Moreover, because of its biological activity of promoting stress tolerance of ECs and being anti-angiogenic, we propose VASH1 to be worthy of further investigation.

Since VASH1 was originally isolated as an angiogenesis inhibitor, our present observation suggests an additional merit of VASH1 [7]. In other words, an increase in the VASH1 level should be beneficial for the prevention or inhibition of angiogenesis-related diseases including cancers. In fact, numerous reports suggested a positive benefit of physical exercise on patients with cancers, but the mechanism of such benefit is ill-defined [19]. We propose an increase in VASH1 as a possible mechanism of such benefit, and this possibility is currently
Processing of VASH1

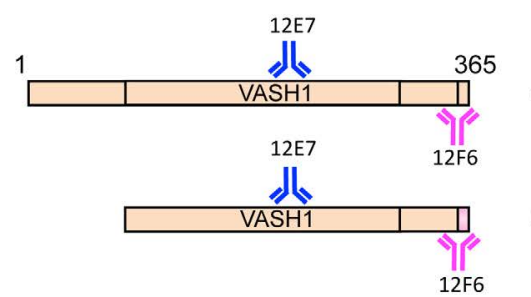

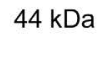

$36 \mathrm{kDa}$

$29 \mathrm{kDa}$
Bioactivity

$+$

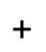

$-$
Detection

by ELISA

O

$\bigcirc$

$\mathrm{X}$
Sandwich ELISA

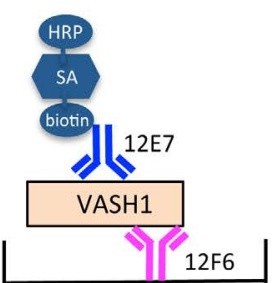

Figure 1. The ELISA used for detection of human VASH1.

Human VASH1 protein is composed of 365 amino acids, and the calculated molecular weight is $44 \mathrm{kDa}$. VASH1 protein is proteolytically processed into $36-\mathrm{kDa}$ and $29-\mathrm{kDa}$ forms after its secretion, and the latter loses its bioactivity. The ELISA for human VASH1 comprised monoclonal antibodies 12F6 against Glu351-Val365 and 12E7 against Ala217-Lys229 for plate coating and horseradish peroxidase (HRP) labeling, respectively. AS: streptavidin. Thus, this ELISA could detect the biologically active forms of VASH1. 


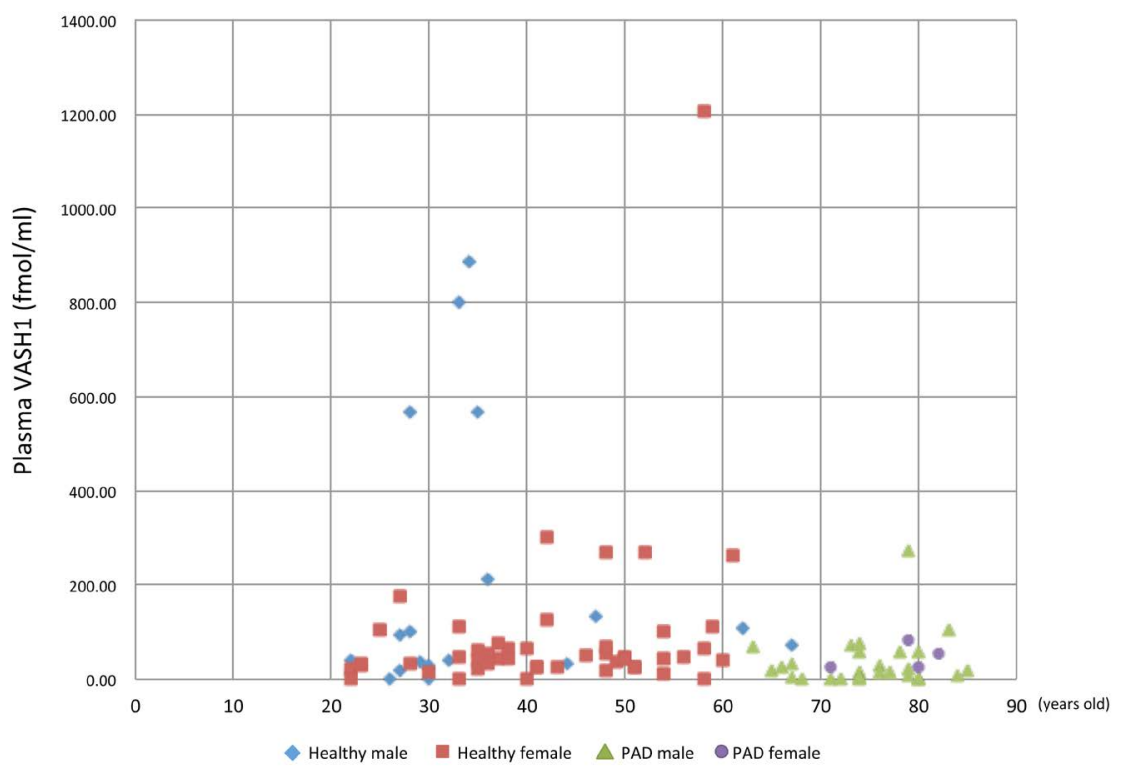

Figure 2. Plasma VASH1 concentrations of all the participants.

Plasma VASH1 concentrations of all the participants are shown in relation to their age.

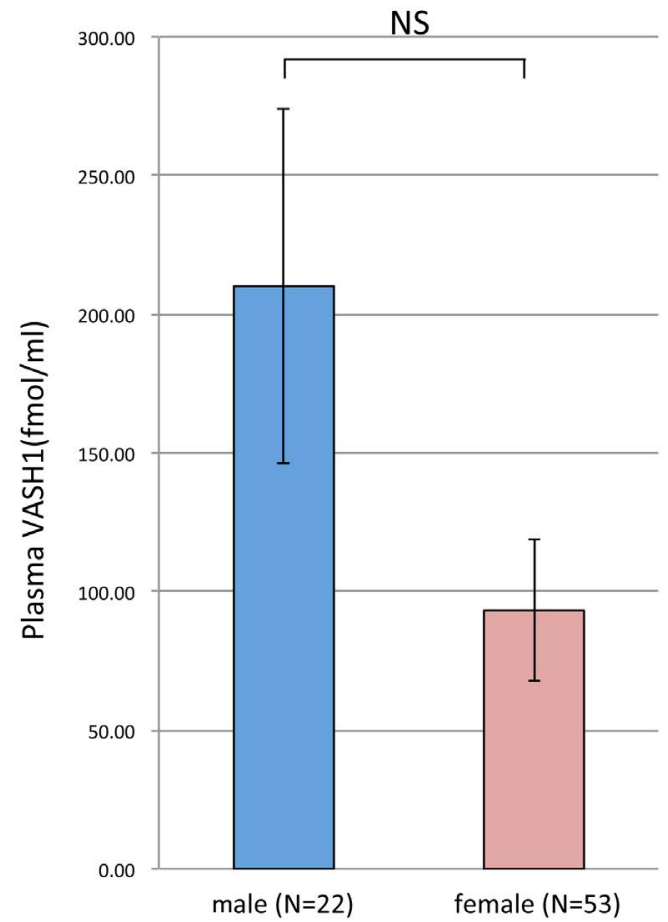

Figure 3. Difference in plasma VASH1 concentration between male and female normal volunteers.

The plasma VASH1 concentration was compared between male $(\mathrm{N}=22)$ and female $(\mathrm{N}=53)$ normal volunteers, but no significant difference was found between them.

under investigation.

In summary, we showed for the first time that physical exercise increased the plasma VASH1 level in elderly patients with peripheral vascular disease. An increase in plasma VASH1 should be beneficial not only for their cardiovascular health but also for the prevention of angiogenesis-related diseases.

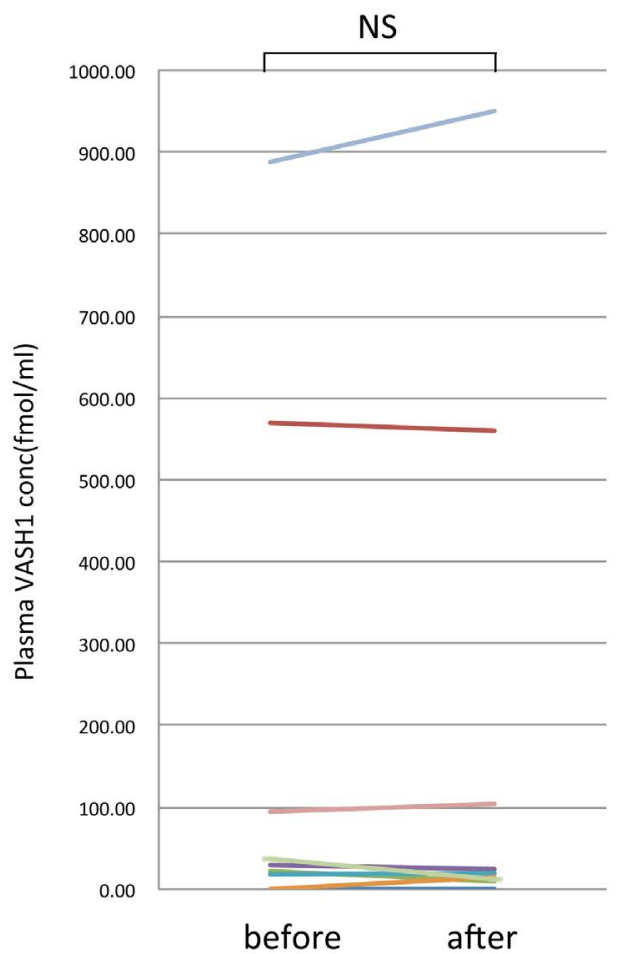

Figure 4. Change in plasma VASH1 concentrations in normal volunteers after acute exercise.

Plasma VASH1 concentrations of 8 normal volunteers were determined before and after acute exercise, but no significant difference was detected.

\section{Acknowledgements}

This work was supported in part by a grant from the Global COE for Conquest of Signal Transduction Diseases with Network Medicine, Tohoku University. 

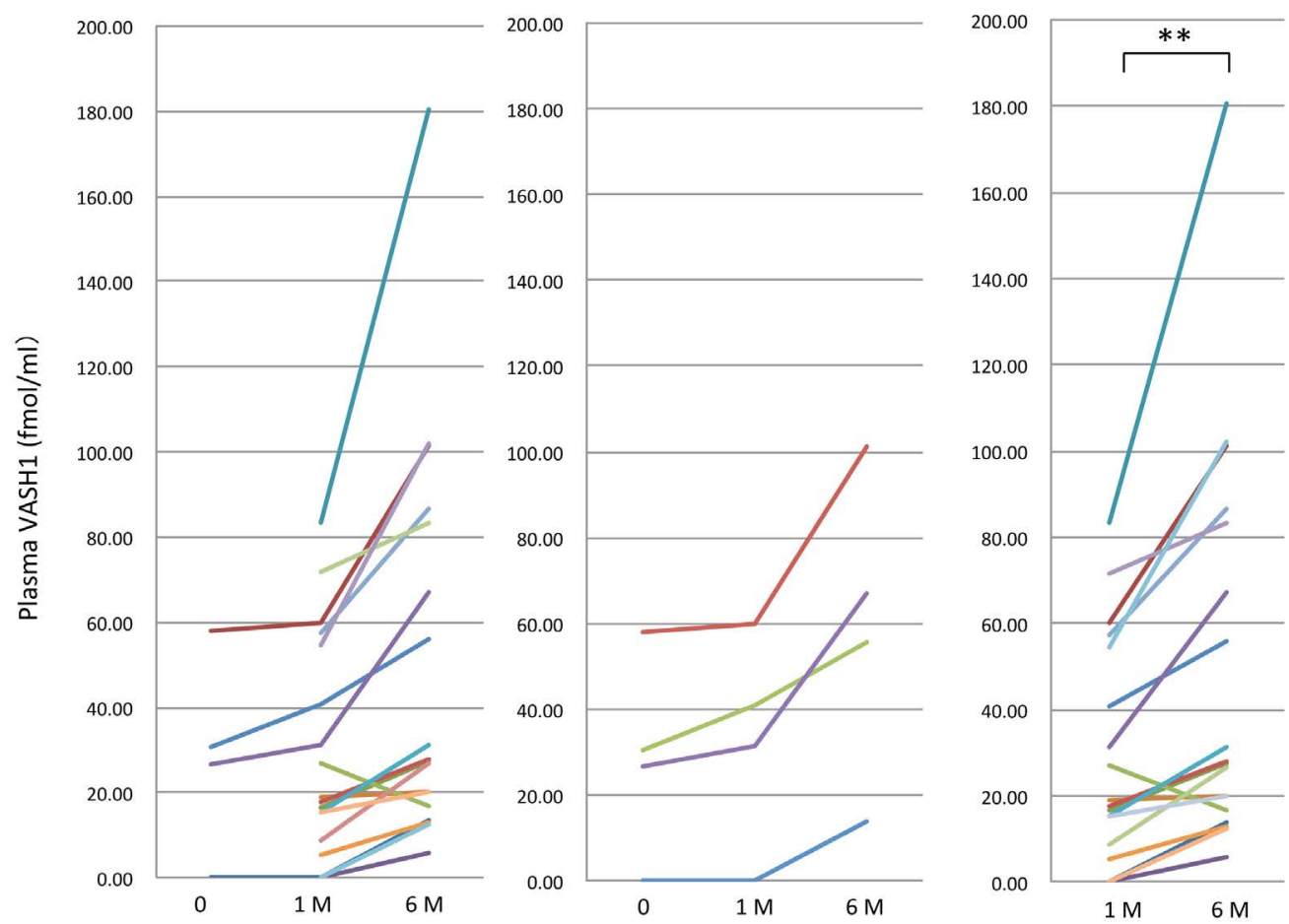

Figure 5. Change in plasma VASH1 level in PAD patients after physical exercise therapy.

Among the 18 patients tested (left graph), we could obtain only 4 patient samples taken before and after 1 month and 6 months of exercise (middle graph). We therefore compared plasma VASH1 levels in 18 patients' samples taken after 1 month and 6 months of exercise (right graph), and found a significant difference between them ("tp $<0.01$ ).

\section{References}

1. Cahill PA, Redmond EM (2016) Vascular endothelium - Gatekeeper of vessel health. Atherosclerosis 248: 97-109. [Crossref]

2. Hazzard WR (1989) Atherosclerosis and aging: a scenario in flux. Am J Cardiol 63: 20H-24H. [Crossref]

3. Costantino S, Paneni F, Cosentino F (2016) Ageing, metabolism and cardiovascular disease. J Physiol 594: 2061-2073. [Crossref]

4. Lavie CJ, Arena R, Swift DL, Johannsen NM, Sui X, et al. (2015) Exercise and the cardiovascular system: clinical science and cardiovascular outcomes. Circ Res. 117: 207-219. [Crossref]

5. Mora S, Cook N, Buring JE, Ridker PM, Lee IM (2007) Physical activity and reduced risk of cardiovascular events: potential mediating mechanisms. Circulation 116: 2110 2108. [Crossref]

6. Sato Y (2015) Novel Link between Inhibition of Angiogenesis and Tolerance to Vascular Stress. J Atheroscler Thromb 22: 327-334. [Crossref]

7. Watanabe K, Hasegawa Y, Yamashita H, Shimizu K, Ding Y, et al. (2004) Vasohibin as an endothelium-derived negative feedback regulator of angiogenesis. J Clin Invest 114: 898-907. [Crossref]

8. Miyashita H, Watanabe T, Hayashi H, Suzuki Y, Nakamura T, et al. (2012) Angiogenesis inhibitor vasohibin-1 enhances stress resistance of endothelial cells via induction of SOD2 and SIRT1. PLoS One 7; e46459. [Crossref]

9. Shimizu K, Watanabe K, Yamashita H, Abe M, Yoshimatsu H, et al. (2005) Gene regulation of a novel angiogenesis inhibitor, vasohibin, in endothelial cells. Biochem Biophys Res Commun. 327: 700-706.

10. Takeda E, Suzuki Y, Sato Y (2016) Age-associated downregulation of vasohibin-1 in vascular endothelial cells. Aging Cell 15: 885-892. [Crossref]

11. Hinamoto N, Maeshima Y, Tanabe K, Nasu T, Saito D, et al. (2014) Exacerbation of diabetic renal alterations in mice lacking vasohibin-1. PLoS One 9: e107934. [Crossref]

12. Heishi T, Hosaka T, Suzuki Y, Miyashita H, Oike Y, et al. (2010) Endogenous angiogenesis inhibitor vasohibin1 exhibits a broad-spectrum anti-lymphangiogenic activity and suppresses lymph node metastasis. Am J Pathol. 176: 1950-1958. [Crossref]
13. Dormandy JA, Rutherford RB, The TASC working group (2000) The treatment of intermittent claudication. $J$ Vasc Surg. 31: S77-89.

14. Sonoda H, Ohta H, Watanabe K, Yamashita H, Kimura H, et al. (2006) Multiple processing forms and their biological activities of a novel angiogenesis inhibitor vasohibin. Biochem. Biophys. Res. Commun. 342: 640-646. [Crossref]

15. Saito M, Suzuki Y, Yano S, Miyazaki T, Sato Y (2016) Proteolytic inactivation of antiangiogenic vasohibin-1 by cancer cells. J Biochem 160: 227-232. [Crossref]

16. Kietadisorn R, Juni RP, Moens AL (2012) Tackling endothelial dysfunction by modulating NOS uncoupling: new insights into its pathogenesis and therapeutic possibilities. Am J Physiol Endocrinol Metab. 302: E481-495. [Crossref]

17. Pinto A, Di Raimondo D, Tuttolomondo A, Buttà C, Milio G, et al. (2012) Effects of physical exercise on inflammatory markers of atherosclerosis. Curr Pharm Des 18: 4326-4349. [Crossref]

18. Lavie CJ, Church TS, Milani RV, Earnest CP (2011) Impact of physical activity, cardiorespiratory fitness, and exercise training on markers of inflammation. $J$ Cardiopulm Rehabil Prev. 31: 137-145. [Crossref]

19. Chyu C, Halnon N2 (2016) Exercise Training in Cancer Survivors. Curr Oncol Rep 18: 38. [Crossref]

Copyright: (C2016 Suzuki Y. This is an open-access article distributed under the terms of the Creative Commons Attribution License, which permits unrestricted use, distribution, and reproduction in any medium, provided the original author and source are credited. 